\title{
A meshless method and its applications in seismic exploration
}

\author{
Xiaofeng Jia, and Tianyue $\mathrm{Hu}$ \\ Dept. of Geophysics, Peking Univ., Beijing, China
}

\begin{abstract}
Wave equation method is one of the fundamental methods for seismic modeling and imaging. In this paper a meshless numerical method - the Element-Free Method (EFM) was applied to solve seismic wave equation and handle modeling and imaging problems. The theory of EFM consisted of two parts - the Moving Least Squares (MLS) criterion and the variational principle, in contrast with the Lagrange interpolation and the variational principle for the theory of the Finite Element Method (FEM). In EFM, it was necessary to calculate the Gauss quadrature on each Gauss point. Only the numbered nodes near to the Gauss point needed to be considered for the quadrature. These nodes were determined by the so-called influence domain of each node. The influence domain was a significant feature of EFM because it effected on the accuracy and cost of the method. At the same time, the absence of elements was also a key point of EFM, which yielded easier preprocessing and lower cost than those of FEM. In this paper, the scheme of EFM would be shown for full scalar or elastic wave equation. Based on the theory, a simple example was discussed in details to indicate the good effectivity of EFM. Furthermore, some synthetic examples will be shown to discover the good performance of EFM in seismic modeling and imaging problems. It is clear that complex structures can be modeled and imaged very well such as high-angle dip and high-velocity anomaly even under complex surface conditions.
\end{abstract}

KEY WORDS: wave equation, EFM, MLS, accuracy, seismic modeling and imaging

\section{INTRODUCTION}

Wave equation method is a well known seismic modeling and imaging method. Quite a few numerical algorithms are developed such as the Finite Difference Method (FDM) and the Finite Element Method (FEM) (Claerbout, 1971; Marfurt, 1984). A meshless method, Element-Free Method (EFM) (Belytschko et al., 1994; Lu et al., 1995), could be a possible solution for seismic wave modeling and imaging. As a matter of fact, the EFM has been demonstrated successful in elasticity, heat conduction and fatigue crack growth problems (Belytschko et al., 1994). In this paper we will apply the EFM to solve seismic wave equation.

In recent years, meshless approximations have become promising methods in solving partial differential equations due to their economy and convenience in applications (Nayroles et al., 1992; Zhu et al., 1998). The EFM is one of these meshless approaches. Only nodal data in the EFM are required in contrast with more knowledge of element connectivity in the FEM. The element mesh is unnecessary. Consequently its preprocessing saves much more time and computer resources than that of the FEM, and the absence of elements gives this method some kind of flexibility as well. In the FEM it is a hard work to determine the shape function due to the complex restraints on it. But in the EFM, these restraints are mostly released, which makes the shape function easily generated with the Moving Least Squares (MLS) criterion. Furthermore, the accuracy of dependent variable in the EFM is no more lower 
than that in the FEM. Indeed, the dependent variable and its gradient are both continuous and therefore post-processing to obtain a smooth gradient field is unnecessary. Based on the theory, this paper will show the applications of the EFM on seismic modeling and imaging.

\section{SOLVING WAVE EQUATION BY EFM}

The following wave propagation problem will be considered in domain $\Omega$ bounded by $\Gamma$ :

$$
\frac{\partial^{2} u}{\partial t^{2}}=D \nabla^{2} u
$$

where $u$ is the displacement field, $D$ is the square of media velocity, and $t, x$ and $y$ denote the time and space coordinates, respectively. The trial function is given by

$$
u^{\mathrm{h}}(x)=p^{\mathrm{T}}(x) a(x)
$$

where $p(x)$ is the basis vector and in the two-dimensional case it is defined by

$$
p^{\mathrm{T}}(x)=[1, x, y] \text { or } p^{\mathrm{T}}(x)=\left[1, x, y, x^{2}, x y, y^{2}\right]
$$

In equation (2) $a(x)$ is an unknown coefficient and it is determined by minimizing the norm as follows:

$$
J=\sum_{I}^{n} w\left(x-x_{l}\right)\left[p^{\mathrm{T}}\left(x_{l}\right) a(x)-u_{I}\right]^{2}
$$

where $u_{I}$ is the nodal value at $x_{I}$ and $n$ is the number of points in the neighborhood of $x$ for which the weight function $w\left(x-x_{l}\right)>0$. This neighborhood is called the influence domain of $x$. The weight function is defined that the further $x_{I}$ is away from $x$, the closer the weight would become to zero. The method of equation (4) is called the MLS fitting criterion.

Substituting $a(x)$ into equation (2), we have

$$
u^{\mathrm{h}}(x)=\varphi(x) U
$$

where $\varphi(x)$ is the shape function vector and $U$ is the nodal displacement vector $\left[u_{1}, u_{2}, \ldots, u_{n}\right]^{\mathrm{T}}$. Equation (5) would be applied to equation (1) using the variational principle. If the penalty Galerkin method is used, the discrete equation under essential boundary condition will be obtained as follows:

$$
K U+M \ddot{U}+F_{\mathrm{q}}=0
$$

where $K$ is stiffness matrix, $M$ is mass matrix and $F_{\mathrm{q}}$ is equivalent load vector. For the time integrator, we use the average acceleration algorithm ( $\mathrm{Lu}$ et al., 1995) so that the time recursion relations in the special case of homogenous boundary condition could be obtained easily and accurately.

The EFM can also be used to treat the problem of elastic wave equation. The equation under consideration is given by

$$
\nabla^{\mathrm{T}} \sigma+b=\rho \ddot{u}
$$

where $\sigma$ is stress vector, $b$ is body force vector, $\rho$ is density of the medium.

Applying equation (5) to equation (7) by penalty Galerkin method will yield the same discrete 
equation as equation (6) except that the expressions of $K, M$ and $F_{\mathrm{q}}$ become more complicated than the above ones. Therefore, the time recursion relations will remain the same as the case of scalar equation.

\section{MODELING VIBRANT FILM BY EFM}

In this section, a numerical example of vibrant film (Jia et al., 2004) will be shown to indicate the good performance of the method. The vibrant film is fixed in the square $0 \leq x \leq 2$ and $0 \leq y \leq 2$. The parameter $D$ in equation (1) is 3. Assuming initial displacement as $u=x(2-x) y(2-y)$ and initial velocity as zero, we can obtain the displacement anytime at any point by solving this two-dimensional scalar wave equation problem.

Both the FDM and EFM are employed to solve this problem. The grid used in the FDM is $33 \times 33$ and in the EFM $21 \times 21$. The time steps for these two methods are $0.002 \mathrm{~s}$ and $0.001 \mathrm{~s}$, respectively. In the EFM, Gauss quadrature has been handled to obtain the matrixes shown in equation (6). The Gauss cells are $20 \times 20$ and in each cell $3 \times 3$ quadrature is employed. For EFM, power function is adopted as the weight function. In fact, other functions such as exponential function and spline function (Belytschko et al., 1994; Dolbow et al., 1999) can also be chosen as the weight, provided that they are characteristic of nonnegativity and monotone decreasing.

The results of some typical nodes are shown in Figure 1 for displacement and Figure 2 for displacement gradient in $x$ direction. Figure 1 and Figure 2 indicate that in addition to good accuracy of displacement, the EFM has especially high accuracy of displacement gradient caused by smoothing effect of MLS fitting. This merit has made the EFM more popular in mechanics than in other fields since the gradient of displacement is usually related to stress and strain.
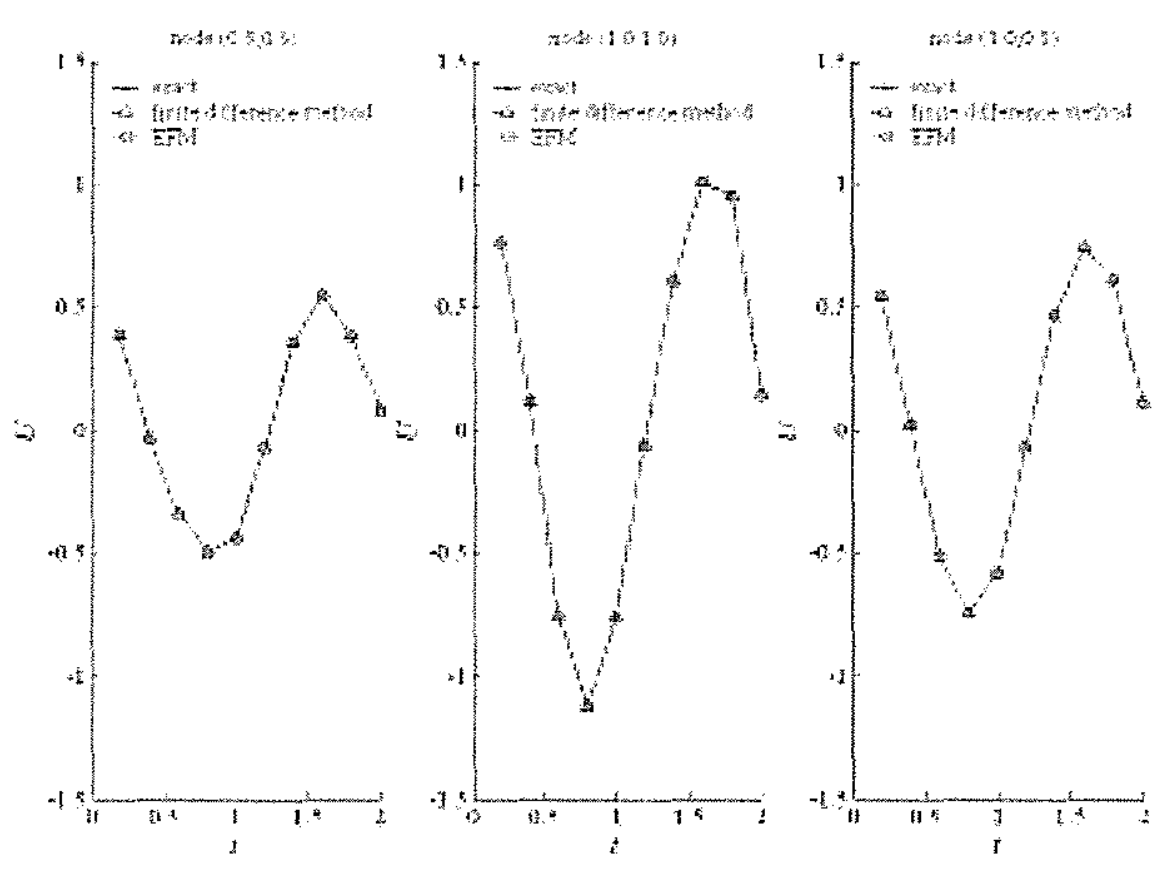

Fig. 1 Modeling the vibrant film: displacement results 

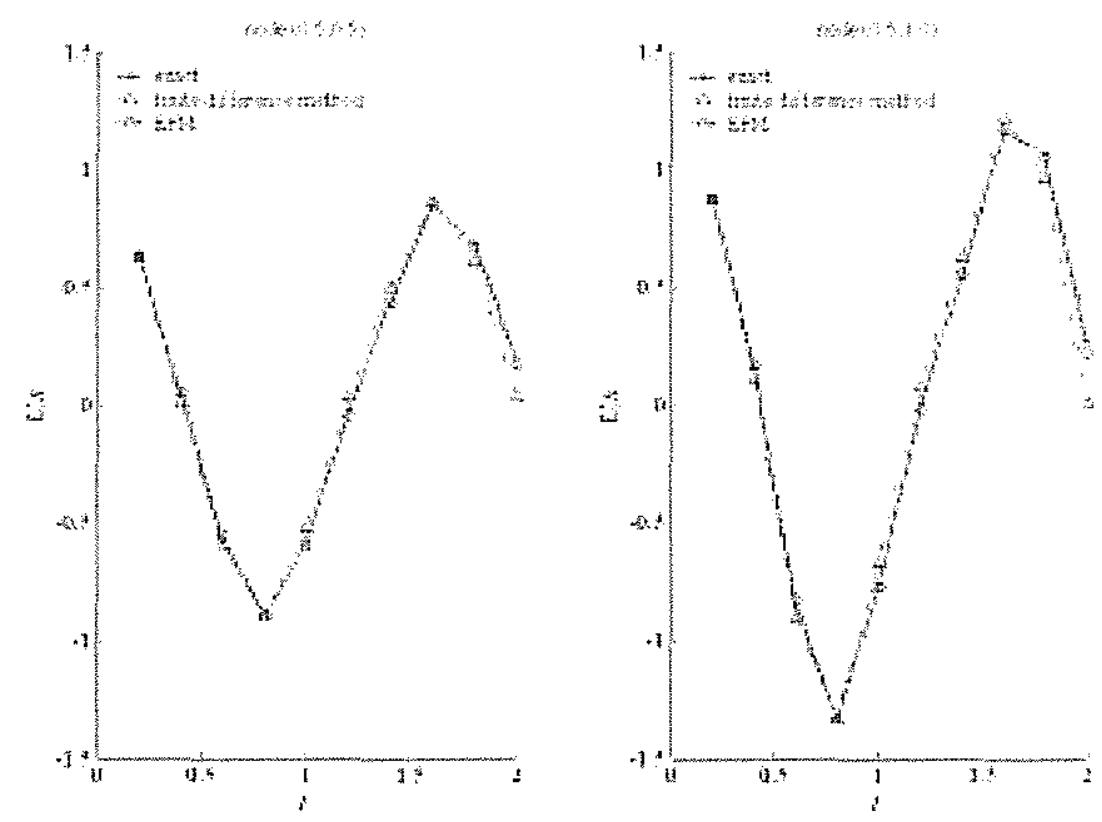

Fig. 2 Modeling the vibrant film: displacement gradient results

\section{SEISMIC MODELING AND IMAGING BY EFM}

As we know, the FEM and the FDM are very common in seismic modeling and imaging. Here it will be proved that the EFM can also process the same problem very well. Three velocity models shown in Figure 3 are designed. For all of them the receivers are distributed evenly on the surface. The first model which includes an embedded high-velocity anomaly and a complex surface structure respectively are used for the case of acoustic poststack modeling and imaging. The second model is designed for the case of acoustic prestack modeling and imaging. The last model is just tested for the elastic prestack modeling problem. The theory of exploding reflector is used to generate poststack seismograms; at the same time, a single split spread is adopted to create prestack seismograms. The sources are sketched in the figure. On the other hand, we use reverse-time migration (McMechan, 1983) to obtain the images. We employ $53 \times 53$ regular nodes for the first two models and $33 \times 33$ nodes for the elastic model. The time step is $0.002 \mathrm{~s}$. For each model $30 \times 30$ Gauss cells and $3 \times 3$ Gauss quadrature are used. The power function is chosen as the weight function.

Figure 4 shows the modeling and imaging results of the first model. In Figure 3(a), $v_{1}=1000 \mathrm{~m} / \mathrm{s}$, $v_{2}=v_{5}=4000 \mathrm{~m} / \mathrm{s}, \quad v_{3}=2000 \mathrm{~m} / \mathrm{s}$ and $v_{4}=8000 \mathrm{~m} / \mathrm{s}$. The paraxial boundary conditions (Clayton et al., 1977) are used to decrease the boundary reflections. The events including primary reflections and residual boundary reflections could be recognized from the seismogram. In the reverse-time migrated section, both the high-velocity anomaly and the horizon near depth $900 \mathrm{~m}$ are imaged very well. The steep layer with its dip over $60^{\circ}$ is also very clear.

In the prestack model shown in Figure $3(\mathrm{~b}), v_{1}=1000 \mathrm{~m} / \mathrm{s}$ and $v_{2}=4000 \mathrm{~m} / \mathrm{s}$. A single shot is exploded $100 \mathrm{~m}$ below the surface. Figure 5 shows its synthetic seismogram and corresponding imaging result. The boundary conditions are handled by the multi-transmitting formula (Liao, 1996). Moreover, the wavefront modeling should be operated to provide the traveltimes for reverse-time migration. Since 
the geometry receives little data from the high-angle dip and the horizon near the geometry side, the image of these structures can hardly be focused.

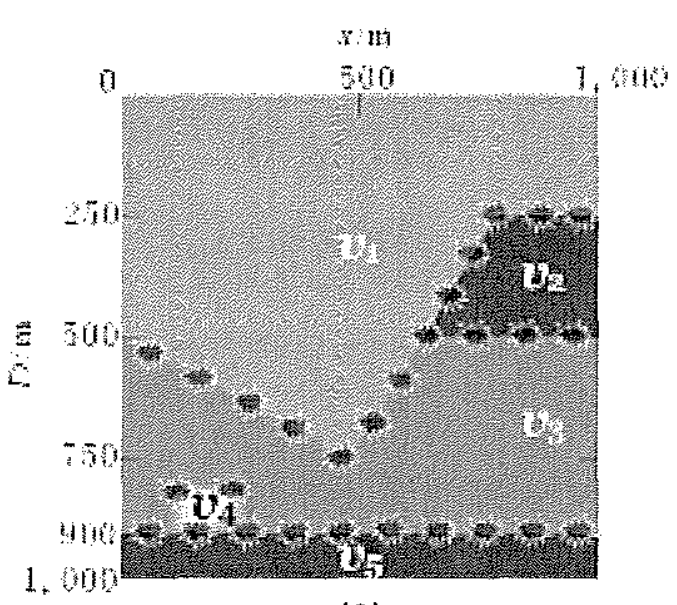

(a)

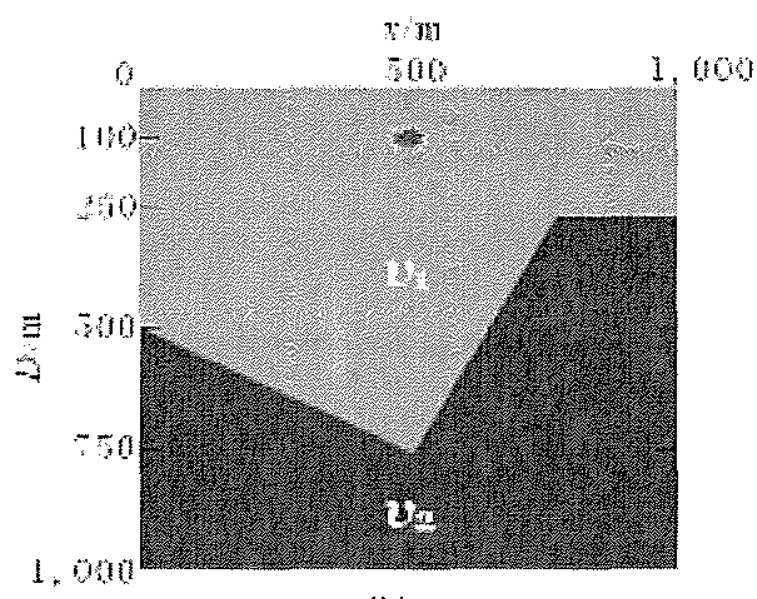

(b)

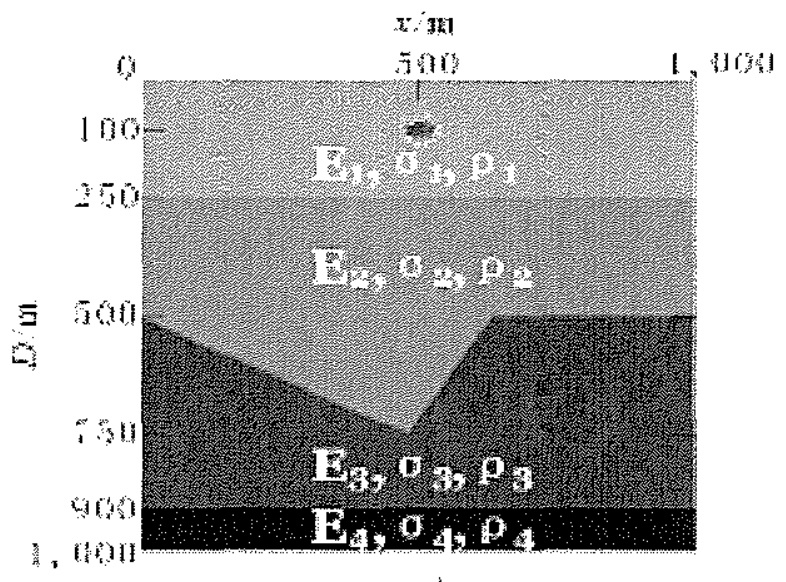

(c)

Fig. 3 Velocity models

Furthermore, the multi-component modeling results for the last model are shown in Figure 6. Medium parameters such as Young's modulus $E$, Poisson ratio $\sigma$ and density $\rho$ in Figure 3(c) are provided as: $E_{1}=14 \times 10^{9} \mathrm{~Pa}, E_{2}=25 \times 10^{9} \mathrm{~Pa}, E_{3}=45 \times 10^{9} \mathrm{~Pa}$ and $E_{4}=60 \times 10^{9} \mathrm{~Pa}$; $\sigma_{1}=0.4, \sigma_{2}=0.25, \sigma_{3}=0.2 \quad$ and $\sigma_{4}=0.1 ; \rho_{\mathrm{l}}=\rho_{2}=2 \times 10^{3} \mathrm{~kg} / \mathrm{m}^{3} \quad$ and $\rho_{3}=\rho_{4}=2.5 \times 10^{3} \mathrm{~kg} / \mathrm{m}^{3}$. Note that the P-wave velocities of the upper two layers are the same while the $\mathrm{S}$-wave velocities are different. This means the multi-component modeling result may indicate the difference between these two layers which is absent in the acoustic modeling result. In fact, from Figure 6 we can find that the reflection occurring on the surface at depth $250 \mathrm{~m}$ has just very little $\mathrm{x}$-component while very marked $\mathrm{y}$-component. Besides the direct waves and primary reflections, the other events such as primary converted reflection could also be tracked. The paraxial wave equations are still used as the absorbing boundary conditions in this case. 

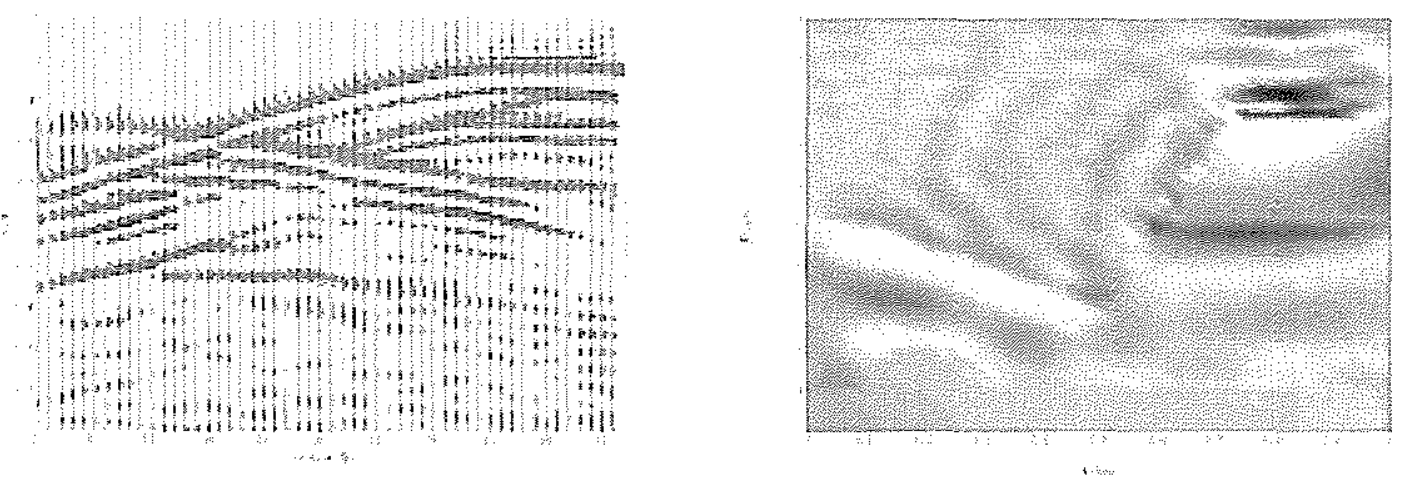

Fig. 4 Poststack modeling (left) and imaging (right) results for model (a)
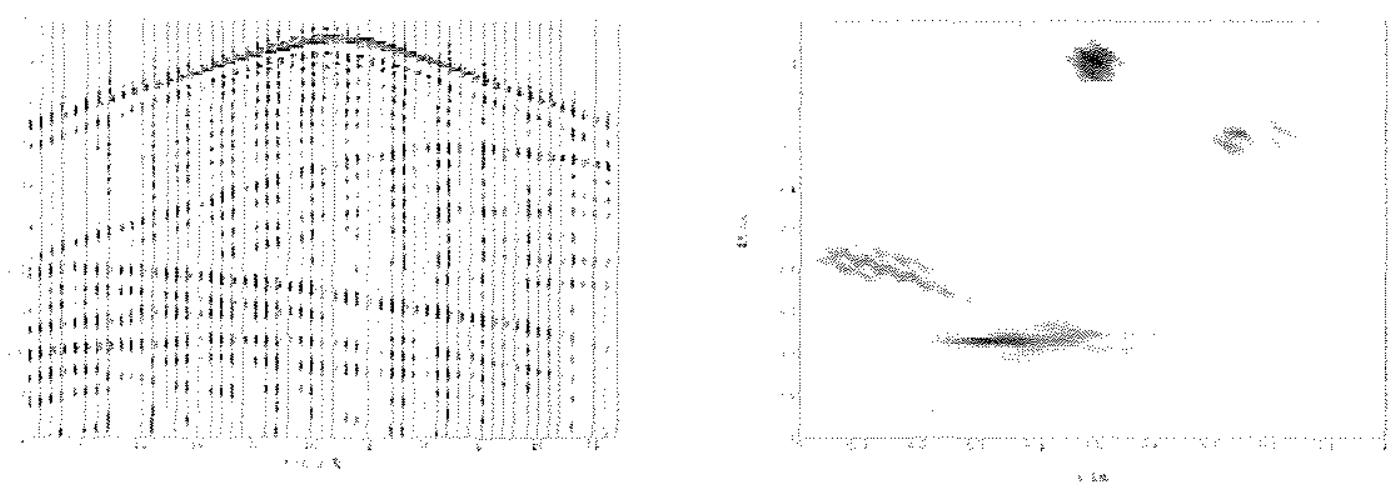

Fig. 5 Prestack modeling (left) and imaging (right) results for model (b)
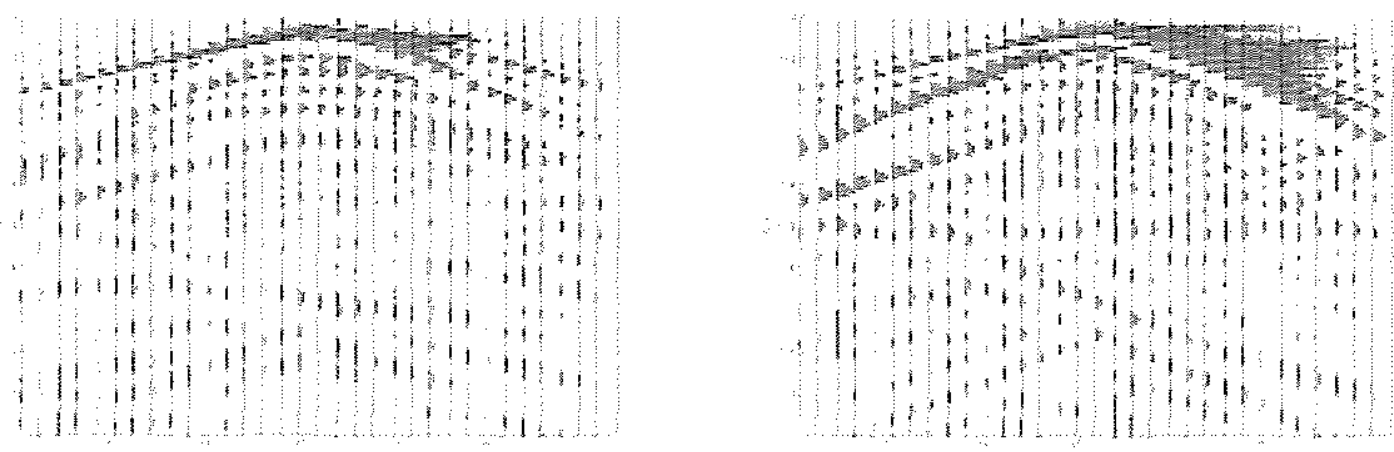

Fig. 6 Prestack modeling results (left $-\mathrm{x}$ component; right $-\mathrm{y}$ component) for model (c)

\section{CONCLUSIONS}

Element-free method, one of the most important meshless methods, has been developed originally in the field of mechanics. In the EFM, the element is unnecessary which saves much computational cost. The influence domain of weight function is also a key feature of the EFM, and one can regard the influence domain as some equivalent element. When enforcing the essential boundary conditions, Lagrange multiplier method, direct boundary collocation and the method using the weak form of the essential boundary conditions are commonly used as well as the penalty method. 
This paper has extended the EFM to seismic wave propagation problems. The results presented here show that the EFM is very promising on seismic modeling and imaging. Even the buried high-velocity anomaly and the structures under complex surface conditions can be modeled and imaged successfully. Note that the imaging results have been obtained in this paper without any prior processing such as noise attenuation on the input data. On the other hand, the advantages of the EFM do not come without any costs. The computation cost is still much higher than the FDM. However, this demerit of cost would become more and more trivial due to the rapid development of computers.

\section{REFERENCES}

Belytschko, T., Lu, Y. Y., and Gu, L., 1994, Element-free Galerkin methods, International Journal for Numerical Methods in Engineering, 37, 229-256.

Claerbout, J. F., 1971, Toward a unified theory of reflector mapping, Geophysics, 36, 467-481.

Clayton, R., and Engquist, B., 1977, Absorbing boundary conditions for acoustic and elastic wave equations, Bull. Seism. Soc. Amer., 67, 1529-1540.

Dolbow, J., and Belytschko, T., 1999, Numerical integration of the Galerkin weak form in meshfree methods, Computational Mechanics, 23, 219-230.

Jia, X., Wang, R., and Hu, T., 2004, Arbitrary difference precise integration method for solving the seismic wave equation, Earthquake Research in China, 18, 36-41.

Liao, Z. P., 1996, Extrapolation nonreflecting boundary conditions, Wave Motion, 24, 117-138.

Lu, Y. Y., Belytschko, T., and Tabbara, M., 1995, Element-free Galerkin method for wave propagation and dynamic fracture, Computer Methods in Applied Mechanics and Engineering, 126, 131-153.

Marfurt, K. J., 1984, Accuracy of finite-difference and finite-element modeling of the scalar and elastic wave equations, Geophysics, 49, 533-549.

McMechan, G. A., 1983, Migration by extrapolation of time-dependent boundary values, Geophysical Prospecting, 31, 413-420.

Nayroles, B., Touzot, G., and Villon, P., 1992, Generalizing the finite element method: diffuse approximation and diffuse elements, Computational Mechanics, 10, 307-318.

Zhu, T., Zhang, J., and Atluri, S., 1998, A local boundary integral equation (LBIE) method in computational mechanics, and a meshless discretization approach, Computational Mechanics, 21 , 223-235. 